MATHEMATICS OF COMPUTATION

Volume 76, Number 260, October 2007, Pages 2161-2179

S $0025-5718(07) 01980-1$

Article electronically published on May 3, 2007

\title{
EFFICIENT CM-CONSTRUCTIONS OF ELLIPTIC CURVES OVER FINITE FIELDS
}

\author{
REINIER BRÖKER AND PETER STEVENHAGEN
}

\begin{abstract}
We present an algorithm that, on input of an integer $N \geq 1$ together with its prime factorization, constructs a finite field $\mathbf{F}$ and an elliptic curve $E$ over $\mathbf{F}$ for which $E(\mathbf{F})$ has order $N$. Although it is unproved that this can be done for all $N$, a heuristic analysis shows that the algorithm has an expected run time that is polynomial in $2^{\omega(N)} \log N$, where $\omega(N)$ is the number of distinct prime factors of $N$. In the cryptographically relevant case where $N$ is prime, an expected run time $O\left((\log N)^{4+\varepsilon}\right)$ can be achieved. We illustrate the efficiency of the algorithm by constructing elliptic curves with point groups of order $N=10^{2004}$ and $N=\operatorname{nextprime}\left(10^{2004}\right)=10^{2004}+4863$.
\end{abstract}

\section{INTRODUCTION}

For an elliptic curve $E$ defined over the finite field $\mathbf{F}_{q}$ of $q$ elements, the order $N=\# E\left(\mathbf{F}_{q}\right)$ of the group of $\mathbf{F}_{q}$-rational points of $E$ is an integer in the Hasse interval

$$
\mathcal{H}_{q}=\left[(\sqrt{q}-1)^{2},(\sqrt{q}+1)^{2}\right]=[q+1-2 \sqrt{q}, q+1+2 \sqrt{q}]
$$

around $q+1$. Various point counting algorithms [24, 21, 14, 11] have been developed over the last 20 years that compute $N$ in polynomial time from the standard representation of $E$ by a Weierstrass equation over $\mathbf{F}_{q}$. A natural 'inverse problem' to the point counting problem is the following.

Problem 1. Given a finite field $\mathbf{F}_{q}$ and an integer $N \in \mathcal{H}_{q}$, find an elliptic curve $E / \mathbf{F}_{q}$ for which $E\left(\mathbf{F}_{q}\right)$ has order $N$.

If $q=p$ is a prime number, then all integers $N \in \mathcal{H}_{p}$ arise as the order of an elliptic curve over $\mathbf{F}_{p}$, and a solution to Problem 1 always exists. For prime powers $q=p^{k}$ this is not generally true: the values $N \in \mathcal{H}_{q}$ having $N \equiv 1 \bmod p$ can only be realized by supersingular elliptic curves over $\mathbf{F}_{q}$, and these are in most cases too rare [26, Theorem V.4.1] to account for all values $N \equiv 1 \bmod p$ in $\mathcal{H}_{q}$. On the other hand, all values $N \not \equiv 1 \bmod p$ in $\mathcal{H}_{q}$ do arise as orders of elliptic curves over $\mathbf{F}_{q}$.

No algorithm is known to solve Problem 1 (in the cases where a solution exists) in a time that is polynomially bounded in the input size $\log q \approx \log N$. Due to the fact that point counting of elliptic curves over $\mathbf{F}_{q}$ can be done in polynomial time, the naive probabilistic algorithm of trying random curves $E / \mathbf{F}_{q}$ until a curve with the right number of points is found has expected run time $\widetilde{O}\left(N^{1 / 2}\right)$. Here we use

Received by the editor November 11, 2005 and, in revised form, June 9, 2006

2000 Mathematics Subject Classification. Primary 14H52; Secondary 11G15.

(C)2007 American Mathematical Society

Reverts to public domain 28 years from publication 
the $\widetilde{O}$-notation to indicate that terms that are of logarithmic size in the main term have been disregarded.

Simple-minded as it is, the naive algorithm compares favorably to the deterministic complex multiplication algorithm to solve Problem 1 that is discussed in the next section. This is due to the size of the auxiliary polynomials ('class polynomials') in that algorithm, which become prohibitively large for most pairs $(q, N)$. In order to obtain algorithms that are substantially better than the naive method, one can relax the conditions in Problem 1 in the following way.

Problem 2. Given an integer $N \geq 1$, find a finite field $\mathbf{F}$ and an elliptic curve $E / \mathbf{F}$ for which $E(\mathbf{F})$ has order $N$.

In the case where the discrete logarithm problem in $E(\mathbf{F})$ is the basis of a cryptosystem, it is important that $N$ has certain properties, e.g., that it is divisible by or equal to a large prime number, whereas the precise value of $q=\# \mathbf{F}$ is less relevant. In this case one needs a solution to Problem 2, not to Problem 1. The observation is not new, and both problems occur in the list of problems in the introduction of [16] that 'can be solved'.

The main result of this paper is that, even though no efficient solution to Problem 1 is known, Problem 2 does admit such a solution if $N$ is provided to the algorithm in factored form. For practical applications, such as those in elliptic curve cryptography, it is unlikely that one will need or want to use elliptic curves for which the factorization of the group order is unknown, so requiring the factorization of $N$ to be part of the input is not a severe restriction. Our solution to Problem 2 for factored orders $N$ is almost polynomial time, provided that one is willing to assume a number of 'standard heuristic assumptions' that we will make explicit in Section 4.

Main Theorem. There exists an algorithm that, on input of an integer $N \geq 1$ together with its factorization, returns a prime number $p$ and an elliptic curve $E / \mathbf{F}_{p}$ with $\# E\left(\mathbf{F}_{p}\right)=N$ whenever such a pair $(E, p)$ exists. Under standard heuristic assumptions, a pair $(E, p)$ exists for all $N$, and the expected run time of the algorithm is polynomial in $2^{\omega(N)} \log N$. Here $\omega(N)$ denotes the number of distinct prime factors of $N$.

Although the run time in the Main Theorem is not polynomial in the usual sense, it is polynomial in $\log N$ outside a zero density subset of $\mathbf{Z}_{>1}$ consisting of very smooth input values $N$. Note that such $N$ are not used in cryptographic applications, as the discrete logarithm problem in groups of smooth order tends to be easy.

Corollary. If the input values $N$ in the Main Theorem are restricted to be prime numbers or, more generally, to be in the density 1 subset of $\mathbf{Z}_{\geq 1}$ consisting of those $N$ having $\omega(N)<2 \log \log N$, then the expected run time is polynomial in $\log N$.

The factorization of $N$ is used by the algorithm in the Main Theorem to reduce square root extractions of small integers modulo $N$ to square root extractions modulo the prime factors of $N$. It is here that the approximate number $2^{\omega(N)}$ of such roots enters the run time of the algorithm. The precise exponents in the run time depend on one's willingness to accept fast multiplication techniques and probabilistic subroutines in the algorithm. For instance, the square root extractions of 
small integers modulo the prime factors of $N$ can be done efficiently by probabilistic means or, much less efficiently, but still in time polynomial in $2^{\omega(N)} \log N$, by a deterministic algorithm [24. Similarly, one may require for the prime number $p$ returned by the algorithm that its primality is proved by a deterministic AKS-type polynomial time algorithm, or employ a faster probabilistic algorithm to do so. If we insist on guaranteed correct output, i.e., a proven prime $p$ as the characteristic of our curve $E$, but allow fast multiplication and probabilistic subroutines of the kind mentioned above, the heuristic run time of our algorithm is $O\left(2^{\omega(N)}(\log N)^{4+\varepsilon}\right)$ for every $\varepsilon>0$ (Corollary 4.4.). In the cryptographically relevant case where $N$ is prime [22, 15], this becomes $O\left((\log N)^{4+\varepsilon}\right)$ (Corollary 4.2).

It should not come as a surprise that our solutions to Problem 2 are elliptic curves defined over prime fields. Indeed, it is easy to see that the union of the Hasse intervals $\mathcal{H}_{q}$ over the prime powers $q$ that are not primes is a zero density subset of $\mathbf{Z}_{\geq 1}$. Solvability of Problem 2 for all values of $N$ is therefore in an informal sense 'equivalent' to the fact that the union of the Hasse intervals $\mathcal{H}_{p}$ over the primes $p$ contains $\mathbf{Z}_{\geq 1}$. Defining the Hasse interval around arbitrary integers $q$ by formula (1.1), we have the equivalence

$$
N \in \mathcal{H}_{q} \Longleftrightarrow q \in \mathcal{H}_{N}
$$

and we see that we want every Hasse interval $\mathcal{H}_{N}$ around an integer $N$ to contain a prime number $p$. This amounts to the statement that the size of the 'gaps' between consecutive primes around $N$ does not exceed $4 \sqrt{N}$. Although prime gaps of this size are not believed to exist, the best proven upper bound on their size [2] is currently $O\left(N^{\alpha}\right)$, with $\alpha=.525>\frac{1}{2}$. Even under assumption of the generalized Riemann hypothesis, the best result [13, Theorem 12.10] is only $O\left(N^{1 / 2} \log N\right)$. This means that we have no proof that Problem 2 is solvable for all $N$, and already for this reason a rigorous run time analysis for our Algorithm is out of reach.

By the prime number theorem, we expect one out of every $\log N$ integers around $N$ to be prime, so the Hasse interval $\mathcal{H}_{N}$ of length $4 \sqrt{N}$ around $N$ will normally contain many primes $p$. In practice, there is always an abundance of primes $p$ for which there exist elliptic curves $E / \mathbf{F}_{p}$ of order $N$, and it seems extremely unlikely that the number of primes in $\mathcal{H}_{N}$, which grows 'on average' as $\widetilde{O}\left(N^{1 / 2}\right)$, will be zero for some $N$. The real task of our algorithm is therefore not so much to find a prime $p \in \mathcal{H}_{N}$, but rather to find a prime $p \in \mathcal{H}_{N}$ for which a curve $E / \mathbf{F}_{p}$ of order $N$ can be constructed efficiently. In Section 2, we show how this leads to a new Problem 3, whose efficient solution yields an efficient solution of Problem 2.

Section 3 describes an algorithm that solves our Problem 3 and finds a suitable prime $p \in \mathcal{H}_{N}$. Its heuristic run time is derived in Section 4. It is based on various unproved but reasonable statements, such as the fact that random integers in $\mathcal{H}_{N}$ will be prime with probability $1 / \log N$. We also present numerical evidence for such unproved statements. In the case where $N$ is prime, the heuristic arguments are very similar to those going into the analysis of the elliptic curve primality proving algorithm ECPP [20].

Section 5 comments on an efficient implementation of the Algorithm to solve Problem 2. It illustrates its practical applicability by treating as examples 'random' values of $N$ such as $N=10^{2004}$ and $N=\operatorname{nextprime}\left(10^{2004}\right)=10^{2004}+4863$. 


\section{Complex multiplication constructions}

Although much in this section generalizes to arbitrary prime powers $q$, we now focus on the case relevant to us, where $q=p>3$ is a prime number and $N \in \mathcal{H}_{p}$ an integer that we want to realize as the order of some elliptic curve $E / \mathbf{F}_{p}$.

Constructing an elliptic curve $E / \mathbf{F}_{p}$ having $N$ points roughly comes down to computing the $j$-invariant $j(E) \in \mathbf{F}_{p}$ of such a curve, and the theory of complex multiplication provides a way of doing so. If we write $N=p+1-t$, then $E / \mathbf{F}_{p}$ has $\# E\left(\mathbf{F}_{p}\right)=N$ if and only if the Frobenius morphism $F_{p}$ of $E$ satisfies the quadratic relation

$$
F_{p}^{2}-t F_{p}+p=0
$$

of discriminant $\Delta=t^{2}-4 p<0$ in $\operatorname{End}(E)$. If $F_{p}$ satisfies $(2.1)$, then $\mathbf{Z}\left[F_{p}\right] \subset$ $\operatorname{End}(E)$ is isomorphic to the imaginary quadratic order $\mathcal{O}_{\Delta}$ of discriminant $\Delta$, and $F_{p}$ corresponds to the element $\pi=\frac{t+\sqrt{\Delta}}{2} \in \mathcal{O}_{\Delta}$ of trace $t$ and norm $p$. Unless we are in the supersingular case $t=0$ having $\Delta=-4 p$, which is too special to be of interest here, this means that $p=\pi \bar{\pi}$ splits into principal primes in $\mathcal{O}_{\Delta}$.

Over the the field $\mathbf{C}$ of complex numbers, it is a classical result that the isomorphism classes of elliptic curves having endomorphism ring isomorphic to $\mathcal{O}_{\Delta}$ correspond to the classes of invertible $\mathcal{O}_{\Delta}$-ideals in the class group $\operatorname{Pic}\left(\mathcal{O}_{\Delta}\right)$ of the order $\mathcal{O}_{\Delta}$. Invertible $\mathcal{O}_{\Delta}$-ideals can be viewed as lattices in $\mathbf{C}$, and the $j$-invariants of these lattices are precisely the $j$-invariants of the elliptic curves having endomorphism ring isomorphic to $\mathcal{O}_{\Delta}$. It follows that we can evaluate these $j$-invariants as values of the modular function $j: \mathbf{H} \rightarrow \mathbf{C}$ in points $\tau_{Q}$ in the complex upper half plane $\mathbf{H}$ representing the ideal classes $[Q] \in \operatorname{Pic}\left(\mathcal{O}_{\Delta}\right)$. More precisely, if we represent the ideal classes of $\operatorname{Pic}\left(\mathcal{O}_{\Delta}\right)$ in the standard way [7, Section 5.2] as reduced binary quadratic forms $Q=a X^{2}+b X Y+c Y^{2}$ of discriminant $b^{2}-4 a c=\Delta$, we have $\tau_{Q}=\frac{-b+\sqrt{\Delta}}{2 a}$. The class polynomial

$$
P_{\Delta}=\prod_{[Q] \in \operatorname{Pic}\left(\mathcal{O}_{\Delta}\right)}\left(X-j\left(\tau_{Q}\right)\right) \in \mathbf{Z}[X]
$$

has integer coefficients, so it can be computed exactly from complex approximations of the $j\left(\tau_{Q}\right)$. In the ordinary case $t \neq 0$, the reduction modulo $p$ of the class polynomial $P_{\Delta}$ splits into $h(\Delta)=\# \operatorname{Pic}\left(\mathcal{O}_{\Delta}\right)$ distinct linear factors in $\mathbf{F}_{p}[X]$, and the roots are the $j$-invariants of the elliptic curves over $\mathbf{F}_{p}$ having endomorphism ring isomorphic to $\mathcal{O}_{\Delta}$. If $j_{0} \neq 0,1728$ is one of these zeroes in $\mathbf{F}_{p}$, then the curve $E_{a} / \mathbf{F}_{p}$ with Weierstrass equation $Y^{2}=X^{3}+a X-a$ has a $j$-invariant $j_{0}$ if we choose $a$ to satisfy

$$
j_{0}=1728 \frac{4 a}{4 a+27},
$$

and its number of points is either $N=p+1-t$ or $p+1+t$. We easily check in which case we are, by point counting or by simply evaluating $N \cdot P$ and $(p+1+t) \cdot P$ for the point $P=(1,1)$ on $E_{a}$. If the order is $N$ we are done; if not, then the quadratic twist $Y^{2}=X^{3}+a g^{2} X-a g^{3}$ of $E_{a}$, with $g$ a non-square in $\mathbf{F}_{p}^{*}$, solves our problem. In the special cases $j_{0}=0,1728$ that we disregard here, there are a few more quadratic twists to consider; see Example 5.2.

Most of the work in the complex multiplication method goes into the computation of the class polynomial $P_{\Delta}$. As the degree of $P_{\Delta}$ and the size of its coefficients both grow like $|\Delta|^{1 / 2}$ for $\Delta \rightarrow-\infty$, the run time can be no better than $\widetilde{O}(|\Delta|)$. 
This is the actual run time [9] for the classical analytic approach using the modular function $j: \mathbf{H} \rightarrow \mathbf{C}$. The same is true for the more recent non-archimedean approach [8, [5], 4] to the evaluation of $P_{\Delta}$, which approximates the roots of $P_{\Delta}$ by a Newton iteration process over $\mathbf{Q}_{\ell}$ for a suitable small prime $\ell$. For both methods, it is possible to reduce the run time by sizable constant factors if one replaces the $j$-function by 'smaller' modular functions [12, 27], 4], 23]. This is very important from a practical, but not from a computational complexity point of view.

In the complex multiplication method, one can save some work by computing the class polynomial $P_{D}$ for the fundamental discriminant $D=\operatorname{disc}(\mathbf{Q}(\sqrt{\Delta}))$ rather than for $\Delta$ itself. As $p=\pi \bar{\pi} \in \mathcal{O}_{\Delta}$ splits in the same way in the maximal order $\mathcal{O}_{D} \supset \mathcal{O}_{\Delta}$ as it does in $\mathcal{O}_{\Delta}$, elliptic curves over $\mathbf{F}_{p}$ with endomorphism ring $\mathcal{O}_{D}$ are just as good for our purposes, and we may everywhere replace $\Delta$ by $D$ in the algorithm. If $\Delta$ has a large square factor, this can be a considerable improvement since the polynomial $P_{D}$ is then much smaller than $P_{\Delta}$.

If we apply the complex multiplication method to solve Problem 1, we have no control over the discriminant

$$
\Delta=\Delta(p, N)=t^{2}-4 p=(p+1-N)^{2}-4 p,
$$

which will typically be of the same order of magnitude as $N$ and without large square factors. In that case, the resulting run time $\widetilde{O}(N)$ is inferior to the $\widetilde{O}\left(N^{1 / 2}\right)$ of the naive probabilistic method.

For Problem 2, the situation is different as only $N$ is then given as input, and we typically have many primes $p \in \mathcal{H}_{N}$ to choose from. An obvious thing to do here is to choose $p \in \mathcal{H}_{N}$ as close as possible to the end points of the interval, so that the absolute value of the trace $t=p+1-N$ differs from $2 \sqrt{p}$ by a small amount. By the prime number theorem, we expect to be able to find $p$ for which $|t|-2 \sqrt{p}$ is of size $\log N$. This makes $\Delta=t^{2}-4 p$ of size $\widetilde{O}\left(N^{1 / 2}\right)$, and reduces the run time of the algorithm to $\widetilde{O}\left(N^{1 / 2}\right)$, just as for the naive probabilistic method.

More generally, one can examine which primes $p$ at distance at most $N^{\alpha}$ from the end points of $\mathcal{H}_{N}$ give rise to values of $\Delta$ with large square factors. Heuristically, there are about $N^{\alpha} / \log N$ such primes, giving rise to discriminants of size $N^{\alpha+1 / 2}$. Among the discriminants of this size, those of the form $\Delta=f^{2} D$ with $|D|<N^{\beta}$ constitute a fraction of order of magnitude

$$
P(\alpha, \beta)=N^{-(\alpha+1 / 2)} \sum_{|D|<N^{\beta} \text { squarefree }} \sqrt{\frac{N^{\alpha+1 / 2}}{|D|}} \approx \operatorname{cst} \cdot N^{\frac{1}{2}(\beta-\alpha)-\frac{1}{4}} .
$$

The number of discriminants $\Delta=f^{2} D$ with $|D|<N^{\beta}$ that we expect to find from $p$ 's no further than $N^{\alpha}$ from the end points of $\mathcal{H}_{N}$ is therefore

$$
P(\alpha, \beta) \cdot \frac{N^{\alpha}}{\log N} \approx \operatorname{cst} \frac{1}{\log N} \cdot N^{\frac{1}{2}(\alpha+\beta)-\frac{1}{4}},
$$

which tends to infinity with $N$ exactly when we have $\alpha+\beta>1 / 2$. Rough as this heuristic analysis may be, it 'explains' why in the example $N=10^{30}$ given in [5. Section 6] to illustrate the non-archimedean approach to computing class polynomials, examining the primes $p$ at distance $<10^{6}$ from the end points of $\mathcal{H}_{N}$ leads to a fundamental discriminant $D \approx-10^{8}$. As examining the primes in an interval of length $N^{\alpha}$ to achieve $|D|<N^{\beta}$ gives rise to a run time $\widetilde{O}\left(N^{\max \{\alpha, \beta\}}\right)$, we can achieve a heuristic run time $O\left(N^{\frac{1}{4}+\varepsilon}\right)$ by taking $\alpha=\beta=\frac{1}{4}+\varepsilon$. Although 
this is still exponential, this method of selecting $p$ already enables us to deal with values of $N$ the naive method cannot handle.

The extreme case $(\alpha, \beta)=(\varepsilon, 1 / 2)$ corresponds to taking $p$ as close as possible to the end points of $\mathcal{H}_{N}$, a case we already discussed. The other extreme $(\alpha, \beta)=$ $(1 / 2, \varepsilon)$ suggests that it should be possible to find $D$ of subexponential size in terms of our input length $\log N$, and that a fruitful approach to solving Problem 2 by the complex multiplication method consists in efficiently minimizing the fundamental discriminant $D$ involved.

It turns out that we can actually determine the 'minimal' imaginary quadratic fundamental discriminant $D$ that can be used to construct an elliptic curve of order $N$ in a relatively straightforward way. It uses the 'symmetry' between the order $N$ of the point group $E(\mathbf{F})$ and the order $q=p$ of $\mathbf{F}$ itself, which are norms of the quadratic integers $1-\pi=1-F_{p}$ and $\pi=F_{p}$, respectively. This symmetry is already familiar to us from (1.2). In the case of the discriminant $\Delta=(\pi-\bar{\pi})^{2}=$ $((1-\pi)-(1-\bar{\pi}))^{2}$ in $(2.2)$, it takes the form

$$
\Delta(p, N)=(p+1-N)^{2}-4 p=(N+1-p)^{2}-4 N .
$$

We now fix $N$ and try to write $\Delta=\Delta(p)$ as

$$
\Delta(p)=(N+1-p)^{2}-4 N=f^{2} D
$$

for 'small' $D<0$. This comes down to solving the positive definite equation

$$
x^{2}-D f^{2}=4 N
$$

in integers $x$ and $f$ in such a way that the number $p=N+1-x$ is prime. This leads us to the following problem.

Problem 3. Given an integer $N \geq 1$, find the smallest squarefree integer $d \geq 1$ together with an algebraic integer $\alpha \in K=\mathbf{Q}(\sqrt{-d})$ such that

(i) $N_{K / \mathbf{Q}}(\alpha)=N$;

(ii) $p=N_{K / \mathbf{Q}}(1-\alpha)=N+1-\operatorname{Tr}_{K / \mathbf{Q}}(\alpha)$ is prime.

The prime $p$ occurring in condition (ii) has the property that there exists an elliptic curve $E / \mathbf{F}_{p}$ having $N$ points and endomorphism ring $\operatorname{End}(E)$ isomorphic to the ring of integers $\mathcal{O}_{K}$ of $K=\mathbf{Q}(\sqrt{-d})$. Once we find the solution $(\alpha, d)$ to Problem 3, we can use it to solve Problem 2 for that same $N$ : take $p=N_{K / \mathbf{Q}}(1-\alpha)$ and construct an elliptic curve over $\mathbf{F}_{p}$ with endomorphism ring $\mathcal{O}_{K}$ for which $1-\alpha \in \mathcal{O}_{K}$ is the Frobenius, using the class polynomial for the order $\mathcal{O}_{K}$. This elliptic curve will have $N=N_{K / \mathbf{Q}}(\alpha)$ points, as desired.

\section{An algorithm to solve Problem 3}

As indicated in the introduction, it is not possible to prove rigorously that any pair $(\alpha, d)$ meeting the conditions of Problem 3 exists at all, let alone that there is a pair with small $d$ that can be found efficiently. We will however argue in the next section why it is reasonable to expect that an integer $d$ solving Problem 3 exists for all $N \geq 1$, and why the smallest such $d$ is even rather small in terms of $N$, of size at most $\widetilde{O}\left((\log N)^{2}+2^{\omega(N)}\right)$. Given this expectation, it makes sense to solve Problem 3 in a straightforward way using an algorithm that, on input of a factored number $N$, tries for increasing squarefree numbers $d \in \mathbf{Z}_{\geq 1}$ to 
- find the integral ideals in $K=\mathbf{Q}(\sqrt{-d})$ of norm $N$;

- determine the generators of those ideals that are principal;

- test for each generator $\alpha$ found whether $N_{K / \mathbf{Q}}(1-\alpha)$ is prime.

As soon as a prime value $p=N_{K / \mathbf{Q}}(1-\alpha)$ is encountered for some $d$, this is the minimal $d$ we are after, and $(\alpha, d)$ is a solution to Problem 3 .

Before we describe an actual algorithm, we look at the three individual tasks to be performed, and the run time of the various subroutines involved. These run times depend on the time $O\left(L^{1+\mu}\right)$ needed to multiply two $L$-bit integers. We have $\mu=1$ for ordinary multiplication, and $\mu=\varepsilon>0$ for the FFT multiplication method. We will give our run times using $\mu=\varepsilon>0$.

Task 1: Finding the integral ideals in $\mathbf{Q}(\sqrt{-d})$ of norm $N$.

Write the ring of integers of $\mathbf{Q}(\sqrt{-d})$ as $\mathbf{Z}[\omega]$, with $\omega=\omega_{d}$ a zero of

$$
f=f_{\mathbf{Q}}^{\omega}= \begin{cases}X^{2}-X+\frac{1+d}{4} & \text { if }-d \equiv 1 \quad \bmod 4 \\ X^{2}+d & \text { otherwise }\end{cases}
$$

Then every ideal of norm $N$ in $\mathbf{Z}[\omega]$ can uniquely be written as $k I$, with $k$ a positive integer for which $k^{2}$ divides $N$, and $I$ a primitive ideal of $\mathbf{Z}[\omega]$ of norm $N_{0}=N / k^{2}$. This last condition means that $\mathbf{Z}[\omega] / I$ is cyclic of order $N_{0}$, and it implies that we have $I=\left(N_{0}, \omega-r\right)$ for some integer $r \in \mathbf{Z}$ satisfying $f(r) \equiv 0 \bmod N_{0}$. Finding all ideals of norm $N$ therefore amounts to finding, for each square divisor $k^{2} \mid N$, the roots of $f$ modulo $N_{0}=N / k^{2}$. It is here that we need to have the factorization of $N$ at our disposal, not only because this implicitly encodes a list of square divisors $k^{2} \mid N$, but also because it enables us to find the roots of $f$ modulo $N_{0}$. Indeed, finding these roots is done by finding the roots of $f$ modulo the prime powers $p^{\operatorname{ord}_{p}\left(N_{0}\right)}$ dividing $N_{0}$, and combining these in all possible ways, using the Chinese remainder theorem, to obtain the roots modulo $N_{0}$. Note that $f$ has no roots modulo $N_{0}$ if $N_{0}$ is divisible by a prime $p$ that is inert in $\mathbf{Z}[\omega]$, or by the square $p^{2}$ of a prime $p$ that ramifies in $\mathbf{Z}[\omega]$.

As finding a root of $f$ modulo an integer essentially amounts to extracting a square root of $-d$ modulo that integer, we need to extract square roots of $-d$ modulo the prime powers dividing $N_{0}$. This easily reduces to extracting square roots of $-d$ modulo each of the primes dividing $N_{0}$. This can be done efficiently by employing a variant of the (probabilistic) Cantor-Zassenhaus algorithm [10, Section 14.5], and leads to an expected run time $O\left((\log p)^{2+\varepsilon}\right)$ to extract square roots modulo a prime $p$. For any selection of square roots $\left(\sqrt{-d} \bmod p^{\operatorname{ord}_{p}\left(N_{0}\right)}\right)$, the Chinese remainder theorem lifts these to a square root modulo $N_{0}$ in time $O\left(\omega(N)(\log N)^{2}\right)$.

Task 2: Finding generators for principal ideals of norm $N$.

For each ideal $k I=k \cdot\left(N_{0}, \omega-r\right) \subset \mathbf{Z}[\omega]$ of norm $N$ found, we use the 1908 algorithm of Cornacchia described in [25, pp. 229-232] or [6] to find a generator of $I$, if it exists. This algorithm performs a number of steps of the Euclidean algorithm to the basis elements $N_{0}$ and $\omega-r$ of the $\mathbf{Z}$-lattice $I=\left(N_{0}, \omega-r\right) \subset \mathbf{Z}[\omega]$ in order to decide whether $I$ is a principal ideal. If it is, a generator $\alpha=k \alpha_{0}$ of $k I$ of norm $N$ is found. For $d \neq 1,3$, the unique other generator of $I$ is $-\alpha$. For the special values $d=1$ and $d=3$ there are 4 and 6 generators for each principal ideal $I$, respectively, obtained by multiplying $\alpha$ by 4 th and 6 th roots of unity. The run time of Cornacchia's algorithm on input $k \cdot\left(N_{0}, \omega-r\right)$ is of order $O\left((\log N)^{2+\varepsilon}\right)$.

Task 3: Testing which algebraic integers $\alpha$ of norm $N$ lead to prime elements $1-\alpha$. 
For each of the elements $\alpha$ of norm $N$ found in step 2, we need to test whether the norm $N+1-\operatorname{Tr}(\alpha)$ of $1-\alpha$ is a prime number. As most $\alpha$ 's will have norms that are not prime, a cheap compositeness test such as the Miller-Rabin test (which takes time $\left.\widetilde{O}\left((\log N)^{2}\right)\right)$ can be used to discard most $\alpha$ 's. Once we find an $\alpha$ for which $N+1-\operatorname{Tr}(\alpha)$ is a probable prime, we do a true primality test to prove primality of $p=N+1-\operatorname{Tr}(\alpha)$. This can be done deterministically in time polynomial in $\log N$ by the 2002 result of Agrawal, Kayal and Saxena [1. Recent speed-ups of the test [18 take time $O\left((\log N)^{6+\varepsilon}\right)$, whereas probabilistic versions [3], 19] have expected run time $O\left((\log N)^{4+\varepsilon}\right)$.

Using the various subroutines specified in the tasks above, we formulate an Algorithm to solve Problem 3. A slightly more practical algorithm that we use to actually find elliptic curves with a given number of points does not exactly follow the outline below; it is discussed in Section 5. The version in this section is phrased to facilitate the heuristic run time estimate in Section 4.

\section{Algorithm.}

Input: a factored integer $N=\prod_{i=1}^{t} p_{i}^{e_{i}}$. Output: a solution $(d, \alpha)$ to Problem 3.

1. Put $d \leftarrow 1$.

2. If $d$ is not squarefree, put $d \leftarrow d+1$ and go to step 2. Otherwise, define $\omega=\omega_{d}$ and $f=f_{\mathbf{Q}}^{\omega}$ as in (3.1).

3. Determine the splitting behavior in $\mathbf{Z}[\omega]$ of all prime divisors of $N$.

3a. Put $k_{1} \leftarrow 1$. For every prime divisor $p_{i}$ of $N$ that is inert in $\mathbf{Z}[\omega]$, put

$$
k_{1} \leftarrow k_{1} p_{i}^{\left\lfloor e_{i} / 2\right\rfloor}
$$

in case $e_{i}$ is even. In case $e_{i}$ is odd, put $d \leftarrow d+1$ and go to step 2 .

3b. For every prime divisor $p_{i}$ of $N$ that ramifies in $\mathbf{Z}[\omega]$, put

$$
k_{1} \leftarrow k_{1} p_{i}^{\left\lfloor e_{i} / 2\right\rfloor} .
$$

4. Put $N_{1} \leftarrow N / k_{1}^{2}$. For every root $\left(r \bmod N_{1}\right)$ of $f$ and for every square divisor $k_{2}^{2} \mid N_{1}$ do the following.

4a. Put $k \leftarrow k_{1} k_{2}$ and $N_{0} \leftarrow N / k^{2}=N_{1} / k_{2}^{2}$. Use Cornacchia to find a generator of $\left(N_{0}, \omega-r\right) \subset \mathbf{Z}[\omega]$, in case it exists.

$4 \mathrm{~b}$. If a generator is found, test for all $(2,4$ or 6$)$ generators $\alpha_{0}$ whether the norm $N+1-\operatorname{Tr}\left(k \alpha_{0}\right)$ of $k \alpha_{0} \in \mathbf{Z}[\omega]$ is prime. If it is, return $d$ and $\alpha=k \alpha_{0}$ and halt.

5. Put $d \leftarrow d+1$ and go to step 2 .

The determination of the splitting behavior of the primes $p_{i} \mid N$ in $\mathbf{Z}[\omega]$ in step 3 amounts to computing the Kronecker symbol $\left(\frac{D}{p_{i}}\right)$ for $D=\operatorname{disc}(\mathbf{Q}(\sqrt{-d}))$. For $p>2$ this is simply the Legendre symbol, which is easily evaluated by combining quadratic reciprocity with the Euclidean algorithm. The factor $k_{1}$ computed in this step is the minimal 'imprimitivity factor' dividing all ideals of norm $N$ in $\mathbf{Z}[\omega]$. It reflects the fact that primitive ideals are not divisible by inert primes, or by squares of ramified primes.

The evaluation of the roots of $f$ modulo $N_{1}$ in step 4 is done by evaluating the roots of $f$ modulo the various prime powers dividing $N_{1}$, and combining these in all possible ways using the Chinese remainder theorem. For the ramified primes $p_{i}$ dividing $N_{1}$, which occur with exponent 1 , there is a unique (double) root of $f$ modulo $p$. For splitting primes $p_{i}$, the polynomial $f$ has exactly 2 different roots 
modulo $p_{i}$, and these lift uniquely to $\mathbf{Z}_{p_{i}}$. Finding the roots of $f$ modulo these $p_{i}$ is non-trivial as it involves the extraction of a square root $\sqrt{-d}$ modulo $p_{i}$. Refining these roots to roots modulo $p_{i}^{e_{i}}$ is much faster, and an easy application of Hensel's lemma. The number of distinct roots modulo $N_{1}$ is $2^{s} \leq 2^{\omega(N)}$, with $s$ the number of $p_{i} \mid N$ that split in $\mathbf{Z}[\omega]$.

Step 4 computes the possible generators of the primitive parts of ideals of norm $N$ in $\mathbf{Z}[\omega]$. It is not completely optimized as it does not take into account that different roots of $f$ modulo $N_{1}$ may coincide modulo $N_{0}$, and give rise to the same ideal $\left(N_{0}, \omega-r\right)$ in step 4a. It also unnecessarily treats the complex conjugate $\left(N_{0}, \omega-r^{\prime}\right)$ of every ideal $\left(N_{0}, \omega-r\right)$, whose generators (if any) are of course the complex conjugates of the generators of $\left(N_{0}, \omega-r\right)$.

\section{HeURistic RUN TIME ANALYSis}

In this section, we present a heuristic run time analysis of the Algorithm in the previous section, and numerical data supporting this analysis.

Assumption 1. For the elements $\alpha=k \alpha_{0} \in \mathbf{Z}[\omega]$ of norm $N$ that we find in step 4b of our Algorithm, the norm of $1-\alpha$ will be an element of the Hasse interval $\mathcal{H}_{N}$ that, apart from being congruent to $1 \bmod k$, does not appear to have any predictable primality properties. Based on the prime number theorem, a reasonable assumption is therefore that for varying $d, r$ and $N_{0}$, the norms found in step $4 \mathrm{~b}$ will be prime with 'probability' at least $1 / \log N$. In other words, the number of times we expect to execute step $4 \mathrm{~b}$ of our Algorithm before we find a prime value is of order of magnitude $\log N$.

Assumption 2. The input for step $4 \mathrm{~b}$ is provided by step $4 \mathrm{a}$, which finds the generators of those ideals of norm $N_{0}$ in $\mathbf{Z}[\omega]$ that are principal. The likelihood for a 'random' ideal in $\mathbf{Z}[\omega]$ to be principal is $1 / h_{d}$, with $h_{d}$ the class number of the ring of integers $\mathbf{Z}[\omega] \subset \mathbf{Q}(\sqrt{-d})$. As we have no indication that the primitive ideals of norm $N_{0}$ arising in step 4a behave differently from random ideals in $\mathbf{Z}[\omega]$, it seems reasonable that they will be principal with 'probability' around $1 / h_{d}$.

The class number $h_{d}$ behaves somewhat irregularly as a function of $d$, but its growth rate $d^{\frac{1}{2}+o(1)}$ was already found by Siegel. In order to bound the number of times we execute steps $4 \mathrm{a}$ and $4 \mathrm{~b}$, we need to bound the integers $d$ we encounter in step 2, i.e., to find an estimate $B_{N}$ for the minimal integer $d$ that occurs in a solution to Problem 3. Clearly, such an estimate will be of heuristical nature, based on the two 'randomness assumptions' above. As our Algorithm consists of a loop over $d=1,2,3, \ldots$, and $d$ has to be factored in step 2 to find if it is squarefree, the value of $B_{N}$ is of great importance in estimating the run time, and the success of our method depends on $B_{N}$ being 'small' as a function of $N$.

Elliptic curves of prime order. In the case our input number $N$ is prime, our Algorithm is similar to the first step of the elliptic curve primality proving algorithm ECPP. On input $N$, this algorithm looks for an imaginary quadratic field $K$ of small discriminant containing an element $\alpha$ of norm $N$ with the property that $N_{K / \mathbf{Q}}(1-\alpha)=N+1-\operatorname{Tr}_{K / \mathbf{Q}}(\alpha)$ is twice a probable prime number $N^{\prime}$. If $\alpha \in K$ is found, $N$ becomes the order of the finite field $\mathbf{F}$ and $2 N^{\prime}$ the number of points of an elliptic curve over $\mathbf{F}$. As $\# \mathbf{F}$ and $\# E(\mathbf{F})$ occur symmetrically in all considerations, this problem is almost identical to our Problem 3. In fact, since finding a prime around a large number $N$ is heuristically just as difficult as finding 
twice a prime around $N$, the heuristic run time for our Algorithm on prime input $N$ is identical to the heuristic run time for the first step of ECPP on input $N$. In accordance with the results in [20, Section 3], we obtain the following.

4.1. Theorem. Let $N$ be a prime number. Under the heuristic Assumptions 1 and 2, the integer d solving Problem 3 is of size $\widetilde{O}\left((\log N)^{2}\right)$, and our Algorithm can be expected to find it in time $O\left((\log N)^{4+\varepsilon}\right)$.

4.2. Corollary. Under the heuristic Assumptions 1 and 2, Problem 2 admits a solution in time $O\left((\log N)^{4+\varepsilon}\right)$ for prime values of $N$.

Proof of Corollary 4.2. We first use our Algorithm to find $d, \alpha$ and $p=N-1+\operatorname{Tr}(\alpha)$ solving Problem 3 for $N$; the time $O\left((\log N)^{4+\varepsilon}\right)$ needed for this dominates the steps that follow. We then construct the class polynomial $P_{D}$ for $D=\operatorname{disc}(\mathbf{Q}(\sqrt{-d}))$ in time $\widetilde{O}(d)=\widetilde{O}\left((\log N)^{2}\right)$. As $P_{D}$ has degree $h_{d} \approx \sqrt{d}$, finding a root $j$ of $P_{d}$ in $\mathbf{F}_{p}$ takes time $\widetilde{O}\left(\operatorname{deg}\left(P_{d}\right)(\log p)^{2}\right)=\widetilde{O}\left((\log N)^{3}\right)$ [10, Section 14.5]. An elliptic curve $E$ with $j$-invariant $j$ and its quadratic twist $E^{\prime}$ will have $N=p+1-\operatorname{Tr}(\alpha)$ or $p+1+\operatorname{Tr}(\alpha)$ points. Matching the group order with the curve can be done efficiently by determining which of the two quantities annihilates random points on the curve. We know that only one of them does for either $E$ or $E^{\prime}$ for all $p>229$ by [25, Theorem 3.2].

Proof of Theorem 4.1. For prime input $N$, our algorithm is rather simple. For increasing values of $d$, it singles out those $d$ for which $N$ is not inert in $\mathbf{Z}\left[\omega_{d}\right]$ in Step 3; in Step 4, it computes the primes over $N$ in $\mathbf{Z}\left[\omega_{d}\right]$ and determines whether these are principal with a generator $\alpha$ for which $1-\alpha$ is a prime element.

The ring $\mathbf{Z}\left[\omega_{d}\right]$ contains elements $\alpha$ of norm $N$ if and only if $N$ splits into principal primes of norm $N$. For primes $N$ coprime to $2 d$, this means that $N$ has to split completely in the Hilbert class field $H_{d}$ of $\mathbf{Q}(\sqrt{-d})$. Our Assumption 2, which states that primitive ideals of norm $N$ should be principal in $\mathbf{Z}[\omega]$ with 'probability' $1 / h_{d}$, now reminds us of the Chebotarev density theorem, which tells us that one out of every $2 h_{d}=\left[H_{d}: \mathbf{Q}\right]$ primes splits completely in $H_{d}$. For $d>3$, it leads us to expect with 'probability' $1 /\left(2 h_{d}\right)$ that there are (up to conjugation) exactly two integral elements $\alpha$ and $-\alpha$ of norm $N$. With complementary probability $1-\left(2 h_{d}\right)^{-1}$, there are no elements of norm $N$. Thus, a value $d$ can be expected to yield an 'on average' number of $1 / h_{d}$ elements of norm $N$.

The average statement that the number of algebraic integers $\alpha \in \mathbf{Q}(\sqrt{-d})$ of norm $N$ is asymptotically a fraction $1 / h_{d}$ of the pairs $(d, N)$ tried is implied by Chebotarev's theorem in case we fix $d$ and let the prime $N$ vary. We are however in the case where $N$ is fixed and $d$ varies. This is certainly different, but for varying $d$ up to a bound $B$ that is small with respect to $N$, it is Assumption 2 that we will find approximately $\sum_{d<B} 1 / h_{d}$ elements of fixed norm $N$. This is reasonable, provided that the fields $H_{d}$ are 'close' to being linearly independent over $\mathbf{Q}$.

It is not exactly true that the Hilbert class fields $H_{d}$ for the squarefree integers $d<B$ we encounter form a linearly disjoint family of number fields: the genus fields $G_{d} \subset H_{d}$ have many non-trivial intersections. However, in this family of fields, which has about $\left(6 / \pi^{2}\right) B$ elements, there is a subfamily of fields $H_{d}$ coming from the prime numbers $d \equiv 3 \bmod 4$ that is linearly disjoint over $\mathbf{Q}$. This follows from the fact that for these primes $d$, the field $H_{d}$ is ramified only at $d$, so every field $H_{d}$ is linearly disjoint from the compositum of the other fields $H_{d}$ in the subfamily. 
As the given subfamily has asymptotically $B /(2 \log B)$ elements, we can treat the family of fields $H_{d}$ with $d<B$ as being linearly independent at the cost of a lower order factor $\log B$ in our estimates. Using the standard estimate $h_{d} \leq \sqrt{d} \log d$ following from the analytic class number formula (cf. [17, formula 2.13]), we see that the sum $\sum_{d<B} 1 / h_{d}$ for squarefree $d<B$ is of order of magnitude at least $(\log B)^{-1} \sum_{0<d<B} \frac{1}{\sqrt{d}} \approx(\log B)^{-1} \int_{0}^{B} \frac{d t}{\sqrt{t}}=2 \sqrt{B} / \log B$.

Taking the factor $\log B$ coming from the lack of linear disjointness into account, we find that for $B$ tending to infinity, Assumption 2 implies that the number of elements of prime norm $N$ coming from $d<B$ is bounded from below by some universal constant times $\sqrt{B} /(\log B)^{2}$. By Assumption 1, we expect to need about $\log N$ elements of norm $N$ in step $4 \mathrm{~b}$. Thus, for prime values $N$ tending to infinity, the size $B_{N}$ of the minimal $d$ solving Problem 3 can be expected to be of size $\widetilde{O}\left((\log N)^{2}\right)$. Note that $B_{N}$ is small with respect to $N$, as required in our heuristical argument.

For the run time of the algorithm, we obtain $O\left((\log N)^{4+\varepsilon}\right)$ exactly as in 20]. The main term in the run time comes from computing $\widetilde{O}\left((\log N)^{2}\right)$ values of $\sqrt{-d}$ $\bmod N$, which each take time $O\left((\log N)^{2+\varepsilon}\right)$, and from proving (as in [3], [19]) that the output is correct, i.e., that we have found $\alpha$ of norm $N$ for which $N+1-\operatorname{Tr}(\alpha)$ is indeed prime.

Numerical support. The table below shows the number of solutions $x, y \in \mathbf{Z}_{\geq 1}$ to the equation $x^{2}+d y^{2}=4 N$ for $d$ ranging over the squarefree integers $d \in[1, B]$ for various $B$. For $N$ we took the 5 primes following $10^{100}$ and $10^{200}$. Note that the spacing of primes around $10^{100}$ and $10^{200}$ is in accordance with Assumption 1.

We see that the growth rate is indeed roughly proportional to $c_{N} \sqrt{B}$, for some constant $c_{N}$ : the numbers double if we quadruple $B$.

The data show that the size of $N$, when large with respect to $B$, is irrelevant: only the classes of the primes over $N$ in the class group of $\mathbf{Z}[\omega]$ are important, not the size of $N$.

\begin{tabular}{l||c|c|c|l}
$\downarrow N \quad B \rightarrow$ & 1000 & 4000 & 16000 & 64000 \\
\hline \hline$p_{1}=10^{100}+267$ & 30 & 57 & 125 & 232 \\
$p_{2}=10^{100}+949$ & 41 & 87 & 161 & 304 \\
$p_{3}=10^{100}+1243$ & 22 & 51 & 93 & 173 \\
$p_{4}=10^{100}+1293$ & 39 & 72 & 145 & 316 \\
$p_{5}=10^{100}+1983$ & 29 & 57 & 123 & 245 \\
$q_{1}=10^{200}+357$ & 46 & 91 & 190 & 354 \\
$q_{2}=10^{200}+627$ & 24 & 51 & 98 & 210 \\
$q_{3}=10^{200}+799$ & 24 & 47 & 90 & 184 \\
$q_{4}=10^{200}+1849$ & 47 & 81 & 170 & 376 \\
$q_{5}=10^{200}+2569$ & 73 & 140 & 275 & 532
\end{tabular}

Figure 1 shows the number of solutions for $p_{2}$ and $p_{3}$. Inspecting the data, we see that the growth rate is indeed close to $\sqrt{B}$. The fluctuation in the graphs is caused by the somewhat irregular behaviour of $h_{d}$. On a logarithmic scale, the graphs do look like straight lines with slope 1/2; see Figure 2.

There are clear differences in the constants $c_{N}$ for various $N$. These can be explained by looking at the contributions coming from composite $d$, which we could 


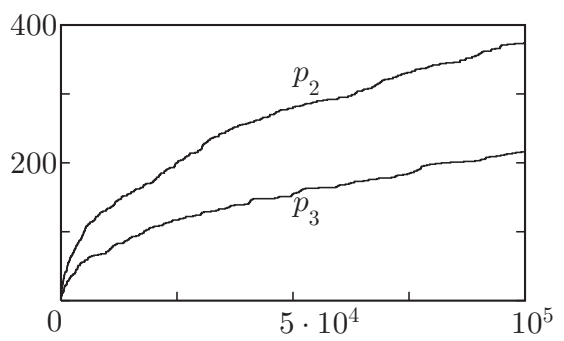

Figure 1

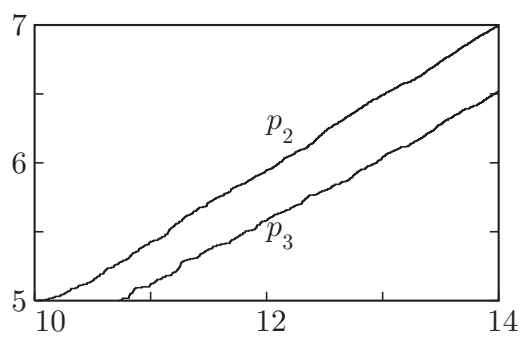

FIGURE 2

afford to neglect in our analysis, but which play an important role in practical situations. For solvability of (2.4), it is clear that $N$ has to be a square modulo all primes dividing $d$. For even $d$, we also have the condition $\left(\frac{N}{2}\right)=1$. If we have $\left(\frac{N}{p}\right)=1$ for many small primes $p$, there will most likely be more composite $d$ yielding solutions to (2.4). The most striking difference in the table occurs for $p_{3}$ and $q_{5}$. Looking at the Kronecker symbols $\left(\frac{q_{5}}{p}\right)$ for the first eight primes $p \leq 20$, we only have $\left(\frac{q_{5}}{p}\right)=-1$ for $p=3,11$. For $p_{3}$ this occurs for $p=2,3,5,13,17$. This explains why $q_{5}$ 'outperforms' $p_{3}$. The differences in the constants $c_{N}$ disappear if we only consider primes $d \equiv 3 \bmod 4$ in our table. For $p_{3}$ we get 53 solutions up to $B=64000$ in this case and for $q_{5}$ we get 50 solutions.

Whereas the number of generators of norm $N$ found in step 4a for $d<B$ increases regularly, and roughly proportional to $\sqrt{B}$, Assumption 1 tells us that the number of times we have to test for primality in step $4 \mathrm{~b}$ before we hit a prime number is $\log N$ on average. As a consequence, we expect that the minimal $d=d(N)$ solving Problem 3 is of size $O\left((\log N)^{2+\varepsilon}\right)$, but not that $d(N)$ increases very regularly with $N$ for prime values $N$. For instance, the primes $p_{1}$ and $p_{5}$ above have rather similar curves exhibiting the number of solutions found in step $4 \mathrm{a}$, but the corresponding minimal discriminants 643 and 303267 are quite far apart: they are the smallest and largest values found for the $p_{i}$. However, the average value of $d$ for the first 100 primes larger than $10^{100}$ and the first 100 primes larger than $10^{200}$ are $82170 \approx\left(\log \left(10^{100}\right)\right)^{2.08}$ and $396030 \approx\left(\log \left(10^{200}\right)\right)^{2.10}$, respectively. Their quotient 4.8 is not too far from the factor 4 we expect.

Elliptic curves of arbitrary order. Assumptions 1 and 2 at the beginning of the section also provide a heuristic run time analysis for arbitrary input $N$.

Assume first that $N$ is squarefree, say $N=\prod_{i=1}^{\omega(N)} p_{i}$ with $p_{i}$ prime. In step 3a, all $d$ are discarded for which one of the primes $p_{i}$ is inert in $\mathbf{Z}\left[\omega_{d}\right]$, so we will only be working in step 4 with those $d$ for which none of the $\omega(N)$ Kronecker symbols $\left(\frac{D}{p_{i}}\right)$ equals -1 . This can be a set of integers of density as small as $2^{-\omega(N)}$ inside the set of all squarefree integers, and in case $N$ is in the zero-density subset of integers satisfying the equivalent inequalities

$$
2^{\omega(N)}>(\log N)^{2} \Longleftrightarrow \omega(N)>\frac{2}{\log 2} \log \log N \doteq 2.88539 \log \log N,
$$

it is clear that we can no longer expect the integer $d$ solving Problem 3 to be of size at most $(\log N)^{2+\varepsilon}$. 
Despite the scarcity of suitable $d$ for large values of $\omega(N)$, it is still the case that we expect the number of elements of norm $N$ coming from $d<B$ to grow at least as fast as some universal constant times $\sqrt{B} /(\log B)^{2}$ if $B$ tends to infinity. Indeed, looking as before at the prime numbers $d \equiv 3 \bmod 4($ not dividing $N$ ) up to $B$, we see that there are ideals of norm $N$ only for a fraction $2^{-\omega(N)}$ of them. However, for each $d$ meeting the $\omega(N)$ quadratic conditions, the number of ideals $I$ of norm $N$ equals $2^{\omega(N)}$ : we can take $I=\prod_{i=1}^{\omega(N)} \mathfrak{p}_{i}$, with $\mathfrak{p}_{i}$ one of the two primes dividing $p_{i}$ in $\mathbf{Z}\left[\omega_{d}\right]$. This means that the growth with $B$ of the number of ideals of norm $N$ coming from $d<B$ is independent of the value of $\omega(N)$ : with increasing $\omega(N)$ they occur for fewer $d$, but the decrease in contributing $d$ is exactly compensated by the number of ideals provided by such $d$. Our expected number of elements of norm $N$ coming from $d<B$ is therefore unchanged with respect to the case of primes $N$ discussed before.

The problem with the asymptotic growth $\sqrt{B} /(\log B)^{2}$ of elements of norm $N$ coming from a thin subset of $d<B$ is that $B$ may have to be large to observe this growth rate: clearly the expected number $2^{-\omega(N)} B$ of contributing $d<B$ should not be too small. As we want to take $B \approx(\log N)^{2}$, we can only use our previous estimate for the expected size of the integer $d$ solving Problem 3 in the case $2^{\omega(N)} \ll(\log N)^{2}$. In the 'opposite' case $2^{\omega(N)} \gg(\log N)^{2}$, finding a single quadratic ring $\mathbf{Z}\left[\omega_{d}\right]$ in which all primes $p_{i} \mid N$ split completely, is what the Algorithm needs to achieve: there will be $2^{\omega(N)}$ ideals of norm $N$ in this ring, of which Assumption 2 tells us we can expect $2^{\omega(N)} / h_{d} \approx 2^{\omega(N)} / \sqrt{d}$ to be principal. As the smallest $d$ satisfying the $\omega(N)$ quadratic conditions imposed by the $p_{i}$ is expected to be of order of magnitude $2^{\omega(N)}$, we will find $2^{\omega(N) / 2} \gg \log N$ elements $\alpha$ of norm $N$ in $\mathbf{Z}\left[\omega_{d}\right]$. By Assumption 1 this will lead to a prime element $1-\alpha$.

4.3. Theorem. Under the heuristic Assumptions 1 and 2, the integer $d$ solving Problem 3 is of size $\widetilde{O}\left((\log N)^{2}+2^{\omega(N)}\right)$, and our Algorithm can be expected to find it in time $O\left(2^{\omega(N)}(\log N)^{4+\varepsilon}\right)$.

4.4. Corollary. Under the heuristic Assumptions 1 and 2, Problem 2 admits a solution in time $O\left(2^{\omega(N)}(\log N)^{4+\varepsilon}\right)$.

Proof of Corollary 4.4. Analogous to the proof of Corollary 4.2.

Proof of Theorem 4.3. We saw that for squarefree $N$, the size of the integer $d$ solving Problem 3 is of size $\widetilde{O}\left((\log N)^{2}\right)$ in case $2^{\omega(N)}$ is of smaller magnitude. If it is bigger, the term $2^{\omega(N)}$ becomes dominant and determines the expected size $\widetilde{O}\left(2^{\omega(N)}\right)$ of $d$.

If $N$ is not squarefree, the Algorithm has an increased number of possibilities to find ideals and elements of norm $N$ for each value of $d$. Primes occurring to even exponents are no longer an obstruction if they are inert in $\mathbf{Z}\left[\omega_{d}\right]$ : they get absorbed in $k_{1}$ in step 3 and no longer occur in $N_{1}$ in step 4 . Splitting primes occurring to higher exponents lead to square divisors $k_{2}^{2} \mid N_{1}$ in step 4 , and to various ideals $\left(N_{0}, \omega-r\right)$ that can be tested for principality in step 4a. The extra ways to find elements of norm $N$ is an advantage, as it will lead to a smaller bound $B_{N}$ for the minimal $d$ solving Problem 3. In particular, $B_{N}$ will be of size $\widetilde{O}\left((\log N)^{2}+2^{\omega(N)}\right)$ for all $N$.

In order to estimate the run time of the Algorithm, we observe that by Assumption 1 , step $4 \mathrm{~b}$ will be executed about $\log N$ times until a probable prime norm is 
found, and a true primality proof taking expected time $O\left((\log N)^{4+\varepsilon}\right)$ is needed. This is the dominant term in the time spent on step $4 \mathrm{~b}$. The number of times Cornacchia's algorithm is executed in step $4 \mathrm{a}$ to yield the $\log N$ generators going into step $4 \mathrm{~b}$ is by Assumption 2 no more than $O\left(\sqrt{B_{N}} \log B_{N} \log N\right)$, as the class numbers $h_{d}$ for $d<B_{N}$ are bounded by $\sqrt{B_{N}} \log B_{N}$. As Cornacchia's algorithm takes time $O\left((\log N)^{2+\varepsilon}\right)$, we expect to spend time $O\left(\sqrt{B_{N}} \log B_{N}(\log N)^{3+\varepsilon}\right)$ in step 4a.

In order to find the roots $\left(r \bmod N_{1}\right)$ of $f$ in step 4 , we first extract the square roots $\sqrt{-d}$ modulo each of the primes $p_{i}$ that split in $\mathbf{Z}\left[\omega_{d}\right]$, in time at most $O\left(\omega(N)(\log N)^{2+\varepsilon}\right)$. For each choice of square roots, there is a root $\left(r \bmod N_{1}\right)$ of $f$ that can be found using the Chinese remainder theorem, in time $\omega(N)(\log N)^{2}$. Each time we apply the Chinese remainder theorem, we use the root $\left(r \bmod N_{1}\right)$ obtained by Cornacchia's algorithm in step 4a. The number of times we apply the Chinese remainder theorem is therefore bounded by the number of times $O\left(\sqrt{B_{N}} \log B_{N} \log N\right)$ we apply Cornacchia's algorithm. We find that the total time spent on finding roots $\left(r \bmod N_{1}\right)$ is no more than $O\left(\sqrt{B_{N}}\left(\log B_{N}\right) \omega(N)(\log N)^{3}\right)$. Taking all parts of step 4 together, the total time spent in step 4 becomes $O\left(\sqrt{B_{N}} \omega(N)(\log N)^{3+\varepsilon}\right)$. This is $O\left((\log N)^{4+\varepsilon}\right)$ for $2^{\omega(N)} \ll(\log N)^{2}$, and it is $O\left(2^{\omega(N) / 2}(\log N)^{4+\varepsilon}\right)$ in general.

Outside step 4, no substantial computing is done, only some administration for the relatively small integer $d$, which takes values up to $B_{N}$. In cases where $B_{N}$ is of order of magnitude $2^{\omega(N)} \gg(\log N)^{2}$, doing this administration is not negligible because of the large number of values taken by $d$. Taking this into account, we find that the heuristic run time is bounded in all cases by $O\left(2^{\omega(N)}(\log N)^{4+\varepsilon}\right)$.

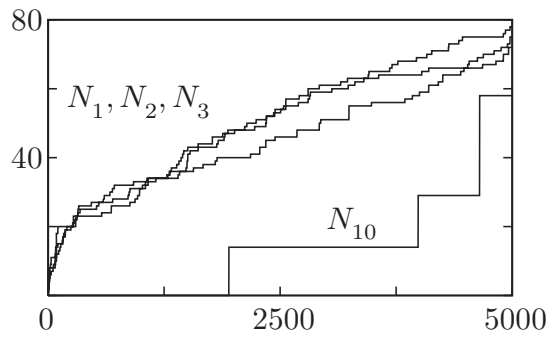

FiguRe 3

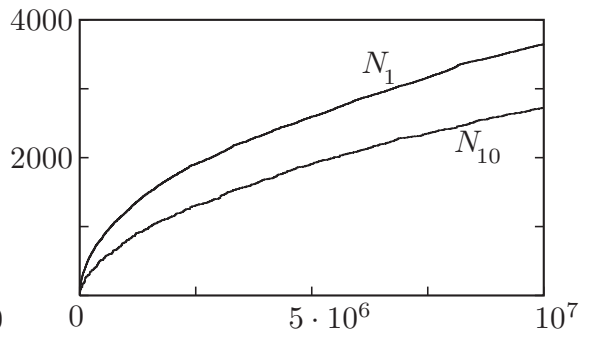

Figure 4 .

Numerical support. Figure 3 shows how the number of solutions $x, y \in \mathbf{Z}_{\geq 1}$ to the equation $x^{2}+d y^{2}=4 N$ for $d$ ranging over all squarefree integers $d \in[1, B]$ varies with $B$ for different values $\omega(N)$ of prime factors of $N$. The graphs are given for $N=N_{1}, N_{2}, N_{3}, N_{10}$, where $N_{k}$ is the product of the first $k$ primes larger than $10^{10}$.

We see that the graphs for $N_{1}, N_{2}$ and $N_{3}$ behave quite similarly. This is what we expected if the number of solutions is independent of $\omega(N)$. The graph for $N_{10}$ appears to be quite different from the others, and this is because $2^{\omega\left(N_{10}\right)}=2^{10}=$ 1024 is of the same order of magnitude as the values of $B$ in the graph. Here there are fewer $d$ for which we have a solution to $x^{2}+d y^{2}=4 N_{10}$, but if we do have a solution, we immediately get many. For instance, the first 'jump' in the graph occurs for the prime value $d=1949$ and we get 28 solutions for this $d$. This is in nice accordance with the heuristics, which tell us to expect the first solutions to occur for $d \approx 2^{10}=1024$, and to be about $2^{5}=32$ in number. The irregularity of the graph for $N_{10}$ disappears if we look at values of $B$ that are large in comparison 


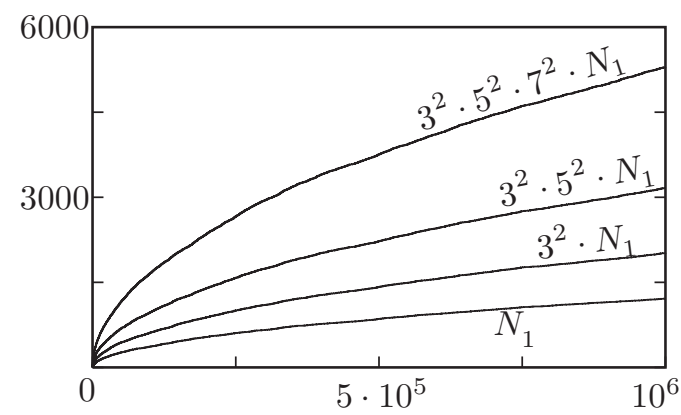

FIGURE 5.

to $2^{\omega\left(N_{10}\right)}$. Figure 4 shows the graph for $N_{10}$ for $B$ up to $10^{7}$. It is now similar in nature to that of $N_{1}$, and exhibits the familiar $\sqrt{B}$-profile.

The graph in Figure 5 illustrates the dependence on the number of square divisors of $N$. It shows the number of solutions for $N_{1}, 3^{2} \cdot N_{1}, 3^{2} \cdot 5^{2} \cdot N_{1}$ and $3^{2} \cdot 5^{2} \cdot 7^{2} \cdot N_{1}$. If $N$ has square divisors, we potentially test the principality of more ideals in step 4 of our Algorithm, so we expect to obtain more solutions. Replacing $N_{1}$ for example by $3^{2} \cdot N_{1}$, we expect to get on average a double amount of solutions for $d \equiv 1$ $\bmod 3$. The gain is a constant factor $>1$ that increases with the amount of square divisors of $N$.

\section{EXAMPLES AND PRACTICAL CONSIDERATIONS}

The description of the Algorithm in Section 3 is intended to facilitate the run time estimate in Section 4, it does not address practical issues that are important in computing large examples. In this section, we explain how we find solutions to Problem 2 for large values of $N$ that are either prime or equal to a power of 10 .

Elliptic curves of large prime order. From the description of the algorithm we gave in the previous section, and more in particular its relation to ECPP, it is clear that one should be able to construct a curve having a large prime number $N$ of points in all cases where ECPP, as described in [20], can prove primality of a number of the same size. To do so, it makes sense to apply an idea attributed to J. Shallit in 20 to speed up the computation. This idea starts from the observation that for large prime numbers $N$, the Algorithm spends a lot of time in evaluating $(\sqrt{-d} \bmod N)$ for all squarefree $d$ up to $B_{N} \approx(\log N)^{2}$ having $\left(\frac{-d}{N}\right)=1$. We noticed already in the previous section that if the equation

$$
x^{2}+d y^{2}=4 N
$$

admits integral solutions, then $N$ is a square modulo all primes dividing $D=$ $\operatorname{disc}(\mathbf{Q}(\sqrt{-d})$. It reflects the fact that if $N$ splits completely in the Hilbert class field $H_{d}$ of $K=\mathbf{Q}(\sqrt{-d})$, then it certainly splits completely in the genus field $G_{d} \subset H_{d}$ of $K$. As $G_{d}$ is obtained by adjoining to $K$ the square roots of $p^{*}=(-1)^{(p-1) / 2} p$ for all odd prime divisors $p \mid d$, we have $\left(\frac{p^{*}}{N}\right)=\left(\frac{N}{p}\right)=1$ in this case.

Once we know that those $d$ providing solutions are essentially products of primes having the right quadratic character with respect to $N$, the idea suggests itself to look at only those $d$ that are constructed as products of such primes. Creating $d$ from a 'basis' of primes $p$ with $\left(\frac{p^{*}}{N}\right)=1$ allows us to compute $\sqrt{p^{*}} \bmod N$ for 
such $p$, and store the values in a list. For $p=2$, one uses the square roots of $-1,2$ and -2 that can be extracted modulo $N$. For each $d$ constructed from our basis of primes, $\sqrt{-d} \bmod N$ can be obtained by multiplying the square roots of primes modulo $N$ we stored. Considering only products of two primes from our basis allows us to reduce the number of square root extractions modulo $N$ from $O\left((\log N)^{2}\right)$ to $O(\log N)$, at the expense of extra multiplications modulo $N$ and an increased storage requirement. In practice, we consider $d$ with at most 3 prime divisors. One thing we lose in this approach is the guarantee that we really find the smallest solution $d$ to Problem 3.

5.1. Example. Take $N=$ nextprime $\left(10^{2004}\right)=10^{2004}+4863$, the exponent 2004 being the year we found our method. For this $N$, we have $\log (N)=4614.3$ and $(\log (N))^{2}=2.13 \cdot 10^{7}$. There are 324 primes $p$ less than 5000 with $\left(\frac{p^{*}}{N}\right)=1$, and we compute and store $\sqrt{2} \bmod N$ and all square roots $\sqrt{p^{*}} \bmod N$. We now have $\left(\begin{array}{c}325 \\ 3\end{array}\right)=5668650$ squarefree values of $d$ at our disposal having up to 3 prime divisors from our base, and we know $N$ to split completely in all genus fields $G_{d}$.

The 104415 -th value of $d$ we tried was $d=59 \cdot 523 \cdot 2579=79580203$. For this value of $d$, we found a solution

$$
x=1885782 \ldots 693127
$$

to $x^{2}+d y^{2}=4 N$ for which

$$
p=N+1-x=999999 \ldots 99999811421 \ldots 8311737
$$

is a 2004-digit prime. In each case, the dots represent 990 digits that we omitted.

The class polynomial $P_{-d}$ has degree 1536 and coefficients up to 41984 digits. Modulo $p$, the polynomial $P_{-d}$ splits completely. Taking $j$ to be the smallest positive integer satisfying $P_{-d}(j) \equiv 0 \bmod p$ we put $a=\frac{27 j}{4(1728-j)} \in \mathbf{F}_{p}$. Then the curve given by

$$
E_{a}: Y^{2}=X^{3}+a X-a
$$

has $\mathrm{CM}$ by $\mathcal{O}_{-d}$. As the point $(1,1) \in E_{a}\left(\mathbf{F}_{p}\right)$ does not have order $N$, the quadratic twist $E_{a}^{\prime}: Y^{2}=X^{3}+9 a X-27 a$ of $E_{a}$ has $N$ points. This can be verified by picking a random point $P \in E_{a}^{\prime}\left(\mathbf{F}_{p}\right)$ and checking that we have $N \cdot P=0$.

The value of $d$ we find here is in fact the smallest $d$ solving Problem 3 for our $N$. Our algorithm did 565 primality tests before we found the solution above. Finding $d$ and $p$ took about 10 minutes on our standard, $2.4 \mathrm{GHz}, \mathrm{PC}$, and another 3 hours were needed to find and factor $P_{-d}$. Once we find $j$, the final result is almost immediate. If we trust the input value $N$ as being a true prime number, there is no need to prove that $p$ is prime. As in ECPP, this follows from the fact that $E_{a}^{\prime}$ has a non-trivial point that is annihilated by $N$.

Elliptic curves of 10-power order. We indicated in our analysis in Section 4 that for input values of $N$ having a large number of square divisors, the integer $d$ solving Problem 3 will be much smaller than the upper bound for squarefree $N$ occurring in Theorem 4.3. This can be illustrated by looking at the values $N=10^{k}$ for $k \geq 1$, which have $\log N \approx 2.3 k$. As none of the prime divisors 2 and 5 of $N$ is inert in the field $\mathbf{Q}(i)$ and the prime 5 is split, there are already many solutions to the norm equation $x^{2}+y^{2}=N$ for the very first value $d=1$. In fact, as we have $h_{d}=1$ there is no need for a Cornacchia algorithm, and the elements of norm $N=2^{k} 5^{k}$ in $\mathbf{Z}[i]$ are the $4 k+4$ elements $\alpha_{s, t}=i^{s}(1+i)^{k}(2+i)^{t}(2-i)^{k-t}$ with 


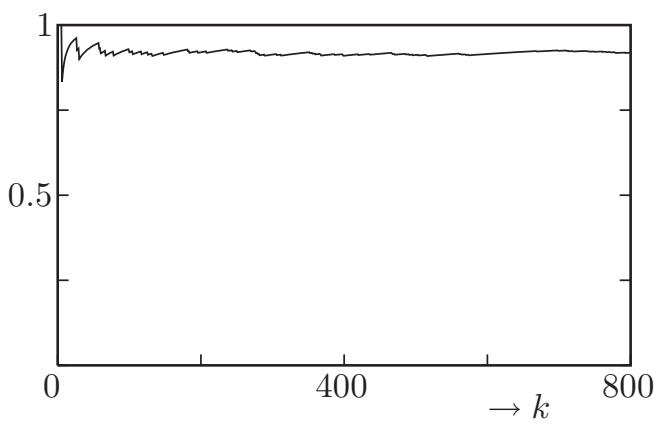

Figure 6.

$s \in\{0,1,2,3\}$ and $t \in\{0,1, \ldots, k\}$. Up to conjugacy, we have about $2 k=.87 \log N$ elements, so we expect that for a positive fraction of all $k$-values, $d=1$ gives rise to a prime $p$ and a twist $E$ of the curve $Y^{2}=X^{3}+X$ having exactly $10^{k}$ points over $\mathbf{F}_{p}$. As the graph in Figure 6 indicates, this fraction appears to be close to 0.92 .

5.2. Example. Take $k=2004$. We find that for $(s, t)=(2,499),(0,527),(0,671)$, the element $\alpha_{s, t}=i^{s}(1+i)^{2004}(2+i)^{t}(2-i)^{2004-t}$ of norm $10^{2004}$ has the property that $p=N_{\mathbf{Q}(i) / \mathbf{Q}}\left(1-\alpha_{s, t}\right)$ is prime. The curve $Y^{2}=X^{3}+X$ having $j=1728$ and CM by $\mathbf{Z}[i]$ has 4 twists over $\mathbf{F}_{p}$ for each of these $p$, but in all cases $Y^{2}=X^{3}+X$ is the curve having $10^{2004}$ points. This follows from a result in 28, going back to Gauss. It says if we choose the prime element $\pi=a+b i$ dividing a prime $p \equiv 1$ $\bmod 4$ in $\mathbf{Z}[i]$ to satisfy $\pi \equiv 1 \bmod (1+i)^{3}$, then the curve $Y^{2}=X^{3}+X$ has exactly $p+1-\left(\frac{-1}{\pi}\right)_{4}(\pi+\bar{\pi})=p+1-2 i^{1-a} a$ points over $\mathbf{F}_{p}$. In our case, $\pi=1-\alpha_{s, t}$ and $a$ are congruent to 1 modulo $(1+i)^{2004}=-2^{1002}$, so we already know that $Y^{2}=X^{3}+X$ is the right curve before actually computing $p$.

For the purpose of constructing curves having $N=10^{k}$ points, there are small values of $d$ that conjecturally work for almost all values of $k$, not just for a positive fraction of them. These $d$ have the property that 2 and 5 both split completely in $\mathbf{Q}(\sqrt{-d})$, i.e., they satisfy $d \equiv 31,39 \bmod 40$. For such $d$, the number of ideals of norm $N$ grows quadratically in $k$, and hence in $\log N$. If we fix $d$, and hence $h_{d}$, the number of elements of norm $N$ in $\mathbf{Q}(\sqrt{-d})$ will also grow quadratically in $\log N$, and our Assumption 2 implies that such $d$ will work for all but finitely many $k$.

5.3. Example. Let $\rho$ be a zero of $X^{3}+X+1$. Then $\rho$ is the value of the Weber function $\mathfrak{f}(z)=\zeta_{48}^{-1} \cdot \frac{\eta\left(\frac{z+1}{2}\right)}{\eta(z)}$ at $-23-1 / \omega_{31}$, and a generator of the Hilbert class field of $\mathbf{Q}(\sqrt{-31})$. An elliptic curve $E_{j} / \mathbf{Q}(\rho)$ having $j$-invariant $j=\left(\rho^{24}-16\right)^{3} / \rho^{24}$ has endomorphism ring $\mathbf{Z}\left[\omega_{31}\right]$. We may take

$$
E_{j}: Y^{2}=X^{3}+3 j(1728-j) X+2 j(1728-j)^{2}
$$

which has good reduction outside $2,3,11,17,23,31$. For all values $1 \leq k \leq 1000$ except $k=1,2$, there exist primes of the form

$$
p=x^{2}+31 y^{2}=10^{k}-1+2 x .
$$

To find them, we write $\left(\omega_{31}+1\right)=\mathfrak{p}_{2} \mathfrak{p}_{5}$ and note that a $\mathbf{Z}\left[\omega_{31}\right]$-ideal

$$
\mathfrak{p}_{2}^{s} \cdot \overline{\mathfrak{p}}_{2}^{k-s} \cdot \mathfrak{p}_{5}^{t} \cdot \overline{\mathfrak{p}}_{5}^{k-t}
$$


of norm $10^{k}$ is principal if and only if we have $s \equiv t \bmod 3$. We use Cornacchia's algorithm to find the generators $\alpha$ for the principal ideals and test whether $N(1-\alpha)$ is prime. For primes satisfying (5.4), either the reduction $\bar{E}_{j} / \mathbf{F}_{p}$ of $E_{j}$ over a prime over $p$ in $\mathbf{Q}(\rho)$ or its quadratic twist has exactly $10^{k}$ rational points over $\mathbf{F}_{p}$. It is likely that $k=1,2$ are the only values of $k$ for which no prime $p$ of the form (5.4) exists, but this is probably very hard to prove.

\section{REFERENCES}

1. M. Agrawal, N. Kayal, N. Saxena, Primes is in P, Annals of Mathematics 160 (2004), 781793. MR2123939 (2006a:11170)

2. R. C. Baker, G. Harman, J. Pintz, The difference between consecutive primes II, Proc. London Math. Soc. (3) 83 (2001), 532-562. MR1851081 (2002f:11125)

3. D. Bernstein, Proving primality in essentially quartic random time, Math. Comp., to appear.

4. R. Bröker, Constructing elliptic curves of prescribed order, Ph.D. Thesis, Universiteit Leiden, 2006. See www.math.leidenuniv.nl/scripties/Broker.pdf.

5. R. Bröker, P. Stevenhagen, Elliptic curves with a given number of points, Algorithmic Number Theory Symposium VI, Springer Lecture Notes in Computer Science, vol. 3076, 2004, pp. 117131. MR2137348 (2005m:11113)

6. J. Buhler, S. Wagon, Basic algorithms in number theory, Surveys in Algorithmic Number Theory, Cambridge University Press, 2006.

7. H. Cohen, A course in computational algebraic number theory, Springer Graduate Texts in Mathematics, vol. 138, 1996. MR1228206 (94i:11105)

8. J.-M. Couveignes, T. Henocq, Action of modular correspondences around CM points, Algorithmic Number Theory Symposium V, Springer Lecture Notes in Computer Science, vol. 2369, 2002, pp. 234-243. MR2041087 (2005b:11077)

9. A. Enge, The complexity of class polynomial computations via floating point approximations, preprint, January 2006.

10. J. von zur Gathen, J. Gerhard, Modern computer algebra, Cambridge University Press, 1999. MR.1689167 (2000j:68205)

11. P. Gaudry, A comparison and a combination of SST and AGM algorithms for counting points of elliptic curves in characteristic 2, ASIACRYPT 2002, Springer Lecture Notes in Computer Science, vol. 2501, 2002, pp. 311-327. MR2087393 (2005h:11124)

12. A. C. P. Gee, P. Stevenhagen, Generating class fields using Shimura reciprocity, Algorithmic Number Theory Symposium III, Springer Lecture Notes in Computer Science, vol. 1423, 1998, pp. 441-453. MR:1726092 (2000m:11112)

13. A. Ivić, The theory of the Riemann Zeta-Function with applications, Wiley, New York, 1985. MR:792089 (87d:11062)

14. K. Kedlaya, Counting points on hyperelliptic curves using Monsky-Washnitzer cohomology, Journal Ramanujan Mathematical Society 16 (2002), 323-338. MR.1877805 (2002m:14019)

15. E. Konstantinou, Y. C. Stamatiou, C. D. Zaroliagis, On the construction of prime order elliptic curves, Progress in cryptology-INDOCRYPT 2003, Springer Lecture Notes in Computer Science 2904, 2003, pp. 309-322. MR2092390 (2005k:14052)

16. G.-J. Lay, H. G. Zimmer, Constructing elliptic curves with given group order over large finite fields, Algorithmic Number Theory Symposium I, Springer Lecture Notes in Computer Science, 1994. MR1322728(96a:11054)

17. H. W. Lenstra, C. Pomerance, A rigorous time bound for factoring integers, J. Amer. Math. Soc. 5 (1992), 483-516. MR.1137100 (92m:11145)

18. H. W. Lenstra, C. Pomerance, Primality testing with Gaussian periods, To appear.

19. P. Mihăilescu, R. M. Avanzi, Efficient 'quasi'-deterministic primalisty test improving AKS, preprint (2003).

20. F. Morain, Implementing the asymptotically fast version of the elliptic curve primality proving algorithm, preprint, arXiv:math.NT/0502097 (2005).

21. T. Satoh, The canonical lift of an ordinary elliptic curve over a finite field and its point counting, Journal Ramanujan Mathematical Society 15 (2000), 247-270. MR.1801221(2001j:11049) 
22. E. Savaş, T. A. Schmidt, Ç. K. Koç, Generating elliptic curves of prime order, Cryptographic hardware and embedded systems - CHES 2001 (Paris), Springer Lecture Notes in Computer Science 2162, 2001, pp. 142-158. MR.1945401(2003m:14037)

23. R. Schertz, Weber's class invariants revisited, J. Théorie des Nombres de Bordeaux 14 (2002), 325-343. MR1926005 (2003j:11139)

24. R. Schoof, Elliptic Curves over Finite Fields and the Computation of Square Roots mod p, Math. Comp. 44 (1985), 483-494. MR777280 (86e:11122)

25. R. Schoof, Counting points on elliptic curves over finite fields, J. Théorie des Nombres de Bordeaux 7 (1995), 219-254. MR1413578 (97i:11070)

26. J. H. Silverman, The arithmetic of elliptic curves, Springer Graduate Texts in Mathematics, vol. 106, 1986. MR817210 (87g:11070)

27. P. Stevenhagen, Hilbert's 12th problem, complex multiplication and Shimura reciprocity, Class field theory - its centenary and prospect, ed. K. Miyake, Adv. studies in pure math., vol. 30, 2001, pp. 161-176. MR 1846457 (2002i:11110)

28. H. P. F. Swinnerton-Dyer, An application of computing to class field theory, Algebraic Number Theory, ed. J. W. S. Cassels \& A. Fröhlich, Academic Press, 1967. MR0219514 (36:2595)

Mathematisch Instituut, Universiteit Leiden, Postbus 9512, 2300 RA Leiden, The NETHERLANDS.

Current address: Department of Mathematics and Statistics, University of Calgary, 2500 University Drive NW, Calgary, Alberta, Canada T2N 1N4

E-mail address: reinier@math.ucalgary.ca

Mathematisch Instituut, Universiteit Leiden, Postbus 9512, 2300 RA Leiden, The NETHERLANDS.

E-mail address: psh@math.leidenuniv.nl 